\title{
Application of Moire Photography to the Study of Dynamic Fracture
}

J. M. HUNTLEY and J. E. FIELD

University of Cambridge, Cavendish Laboratory, Madingley Road, Cambridge CB3 OHE, UK

\section{ABSTRACT}

The use of moiré photography as a technique for measuring the dynamic displacement field, and dynamic stress intensity factor $\left(K_{\mathrm{Id}}\right)$ of a fast crack has been investigated. Sequences of double exposure moiré photographs of cracks moving through plates of polymethylmethacrylate (PMMA) were recorded with a rotating mirror camera at $2 \mu \mathrm{s} \mathrm{frame}^{-1}$. One sequence was analysed in detail to reveal the time variation of both in-plane displacement components, and $\mathrm{K}_{\mathrm{Id}}$. The paper ends with a description of a high resolution moire apparatus, capable of resolving 150 lines $\mathrm{mm}^{-1}$ preliminary results from quasi-statically loaded cracks are presented.

\section{KEYWORDS}

Dynamic fracture; dynamic stress intensity factor, moiré photography; automatic fringe analysis.

\section{INTRODUCTION}

The measurement of the dynamic stress intensity factor $\left(\mathrm{K}_{\mathrm{Id}}\right)$ at the tip of a fast crack has been the subject of considerable research activity over recent years. Optical techniques which have been applied to the study of dynamic fracture include the method of caustics (Manogg, 1964; Theocaris and Gdoutos, 1972), phos5) ( established as the two main techniques for measuring $\mathrm{K}$. However, a transparent materia exhibiting stress-induced bircfringence is required for photoelastic work, and the method of caustics provides only limitcd information on the stress state near the crack tip. Furthermore, photoelasticity and the method of caustics do not always produce consistent results (Dally, private communication). There is a clear need for improved experimental techniques, in order to resolve important questions such as the uniqueness of the relationship between crack velocity and $\mathrm{K}_{\mathrm{Id}}$, and mechanisms for crack branching. For these reasons the technique of moire photography has bee investigated as an alternative method for determining $\mathrm{K}_{\mathrm{Id}}$

\section{DYNAMIC MOIRE PHOTOGRAPHY}

In experimental mechanics, the moiré technique refers to the superimposition of two line gratings: one attached to the specimen (specimen grating), the other stationary (reference grating). A fringe one attached the specimen (specimen grating), the other the in-plane displacement componen pattern is formed which is essentially a contour map to specimen grating pitch. When both 
in-plane components are required, a crossed specimen grating is used; the two fringe patterns 列 $\mathrm{x}$ and $\mathrm{y}$ axes are called the $\mathrm{u}_{\mathrm{x}}$ - and $\mathrm{u}_{\mathrm{y}}$-field patterns, respectively. Further details of the standard moire technique are described by Sciammarclla (1982).

The application of moiré to dynamic events is not straightforward, however. Firstly, there is insufficient time to rotate the line reference grating through the $90^{\circ}$ needed to separate the $u_{x}-$ and $\mathrm{u}_{\mathrm{y}}$-field patterns. This problem can be avercome by recording images of the specimen grating with a high speed camera, the patterns are then separated by optical spatian 1982). The drawback now is that the camera must be able $10-30$ line $\mathrm{mm}^{-1}\left(\mathrm{l} \mathrm{mm}^{-1}\right)$ in the camerast magnificd resulting in a basic trade-off between displacement accuracy (determined by the mecimen grating pe less than the specimen grating pitch divided by maximum specimen velocity, otherwise the image of the grating will be blurred. In this section, results are presented from a series of high speed photographic sequences of fast crack growth in polymethylmethacrylate (PMMA). In the next section, it is shown how the trade-off between grating pitch and field of view can be eliminated by forming the moire fringe patterns outsile the camera.

For the experiments described in this section, a Beckman and Whitley model 189 rotating mirro camera was used on account of its good spatial and temporal resolution. The specimen grating frequency was $40 l \mathrm{~mm}^{-1}$, although the claned resolution of the camera is over $30 l \mathrm{~mm}^{-1}$, in practice it was necessary to magnify by about 2.5 times, resulting in an image plane frequency of about $16 l \mathrm{~mm}^{-1}$, and a field of view of $10 \times 6 \mathrm{~mm}^{2}$. The specimen grating was a commercially available stripping film, attached to the specimens by cyanoacrylate adhesive. The specimens were made from cast PMMA (ICl 'Perspex') to dimensions of $40 \times 32 \times 3 \mathrm{~mm}^{3}$ (see Fig. 1(a)). A

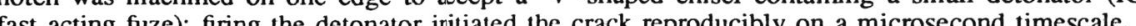

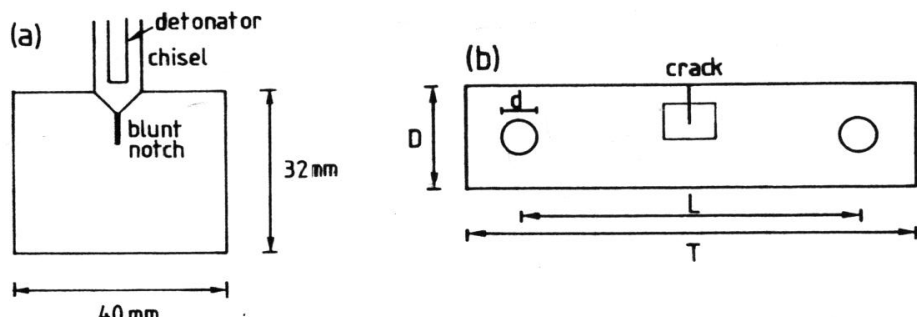

$40 \mathrm{~mm}$ Fig. 1. Specimen geometries for (a) dynamic and (b) quasi-static
experiments. Specimen dimensions shown in $1(\mathrm{~b}): \mathrm{T}=305 \mathrm{~mm}$; $\mathrm{L}=229 \mathrm{~mm} ; \mathrm{D}=76 \mathrm{~mm} ; \mathrm{d}=25.4 \mathrm{~mm}$

A total of seven double exposure sequences were recorded by transmitted light. The film used was A toral the first exposures were made by running the camera up to speed and firing the flash. The specimen was then loaded to about hilf the failure stress, and the magnification was changed by about $2 \%$ to produce sufficient mismach fringes in the final moiré photographs. The second run of the camera fired the detonator and recorded the deforming grid associated with the propagating crack.

Figure 2(a) shows one such doubly exposed frame from one of the sequences, recorded a $2 \mu$ s frame ${ }^{-1}$. Subsequent spatial filtering reveals the $u_{x}{ }^{-}$and $u_{y}-$ field fringes at high contrast (Figs. 2(b) and (c)). The u pattem is supeior because the camera construction results in better spatio resolution for vertical lines than for horizontal lines. The slightly speckled appearance of the tinges is a result of scattering by the film. Normally when spatially filtering, a much finer grained film would be used; such a film would have been too slow for this application, however. The patterns were analysed manually, by converting the fringe orders to displacement components on was then subtracted to give the experimentally observed displacement field resulting from the

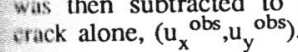

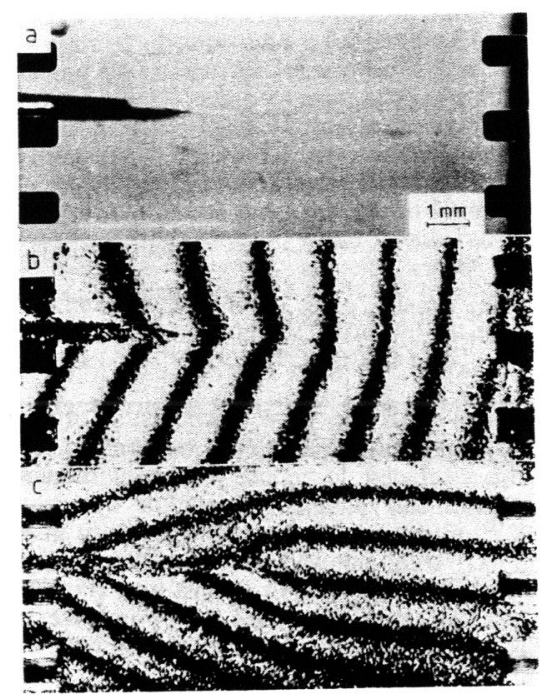

Fig. 2(a). Single frame from sequence of double cxposure moire phot phe of $2(a)$, revealing the $u_{x}$ - and $u_{y}$-ficld fringes, respectively.

\section{Least-squares analysis}

The dynamic stress intensity factor, $\mathrm{K}_{\mathrm{Id}}$, was estimated by minimising the sum

$$
\mathrm{S}^{2}=\sum_{\mathrm{i}=1}^{\mathrm{N}}\left(\left(\mathrm{u}_{\mathrm{x}}^{\text {calc }}-\mathrm{u}_{\mathrm{x}}{ }^{\text {obs }}\right)^{2}+\left(\mathrm{u}_{\mathrm{y}}{ }^{\text {calc }}-\mathrm{u}_{\mathrm{y}}{ }^{\text {obs }}\right)^{2}\right)
$$

over $\mathrm{N}$ datapoints. The superscript calc refers to the theoretical dynamic displacement field. In a coordinate system $(x, y)$ moving such that the origin is always at the crack tip, and with the crack the I displacements can be expressed as a sum of (Nishioka and Atluri, 1983):

$$
\begin{aligned}
& u_{x n}=\left[K_{n}{ }^{o} B(a)(n+1) /(2 \pi)^{1 / 2} \mu\right]\left[r_{1}{ }^{n / 2} \cos \left(n \theta_{1} / 2\right)-h(n) r_{2}{ }^{n / 2} \cos \left(n \theta_{2} / 2\right)\right] \\
& u_{y n}=\left[K_{n}{ }^{o} B(a)(n+1) /(2 \pi)^{1 / 2} \mu\right]\left[-\mathrm{s}_{1} r_{1}{ }^{n / 2} \sin \left(n \theta_{1} / 2\right)+\left(h(n) / s_{2}\right) r_{2}{ }^{n / 2} \sin \left(n \theta_{2} / 2\right)\right]
\end{aligned}
$$


where

$$
\begin{aligned}
& \mathrm{s}_{1}^{2}=1-\mathrm{a}^{2} / \mathrm{c}_{1}^{2} \\
& r_{1}^{2}=x^{2}+s_{1}^{2} y^{2} \\
& \tan \theta_{1}=\mathrm{s}_{1} \mathrm{y} / \mathrm{x} \\
& c_{1}^{2}=(\kappa+1) \mu /(k-1) \rho \\
& B(\mathrm{a})=\left(1+\mathrm{s}_{2}{ }^{2}\right) /\left(4 \mathrm{~s}_{1} \mathrm{~s}_{2}-\left(1+\mathrm{s}_{2}{ }^{2}\right)^{2}\right) \\
& \mathrm{h}(\mathrm{n})=2 \mathrm{~s}_{1} \mathrm{~s}_{2} /\left(1+\mathrm{s}_{2}{ }^{2}\right) \\
& =\left(1+\mathrm{s}_{2}^{2}\right) / 2 \\
& \kappa=(3-v) /(1+v) \\
& =(3-4 v)
\end{aligned}
$$

$a$ is the crack speed, $\rho$ the density, $\mu$ the shear modulus, and $v$ Poisson's ratio. The parameters $K_{n}{ }^{\circ}$ represent the contribution of the nth eigenfunction $(n=0,1,2,3 \ldots)$ to the stress field; the singular term corresponds to $n=1$, and $K_{I d}$ is $K_{1}{ }^{\circ}$.

Datapoints within $1.5 \mathrm{~mm}$ (half the plate thickness) of the crack tip were excluded from the analysis because of 3-dimensional effects (Yang and Freund, 1985), and points more than $2.5 \mathrm{~mm}$ from the tip were also excluded to reduce boundary influences. Plane stress conditions were assumed to prevail within this annulus of eligible datapoints. In the analysis, the first two and $\Delta y$ ) and rotation $(\Omega)$ were allowed to vary. The average crack velocity was measured as $380 \pm 30 \mathrm{~m} \mathrm{~s}^{-1}$ from a plot of crack length against time (Fig. 3(a)). The loading time for a point a distance $\mathrm{r}$ from the crack tip is $\tau \approx \mathrm{r} / \mathrm{a}$ a around $10^{-5} \mathrm{~s}$ in these experiments. The appropriate values for Young's modulus, E, and Poisson's ratio, v, on this timescale are $5.65 \mathrm{GPa}$ and 0.34 , respectively (Read and Dean, private communication).

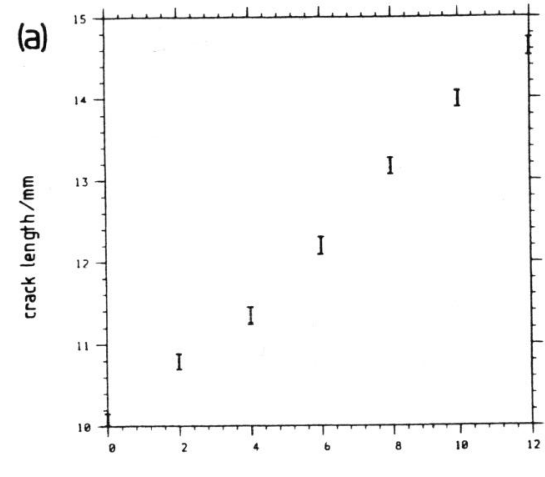

time / ps

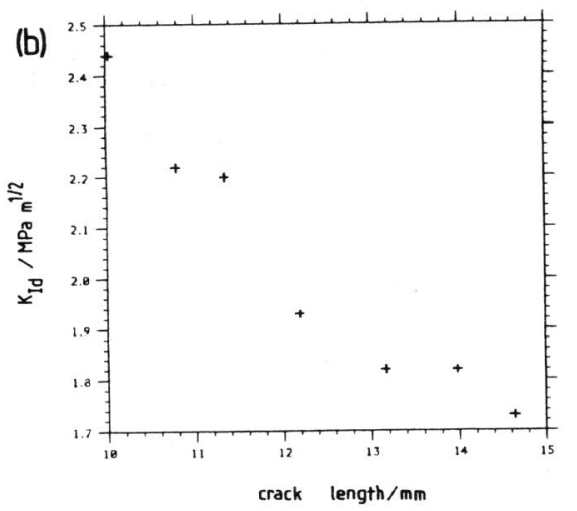

Fig. 3. (a) Crack length as a function of time. The total length of the blunt starter notch (including the ' $\mathrm{V}$ ' notch) was $10.0 \mathrm{~mm}$. (b) $\mathrm{K}_{\mathrm{Id}}$ as a function of crack length, as calculated by a lincar least squares method from the moire fringe patterns.

Seven frames were analysed in this way, and the results are plotted in Fig. $3(\mathrm{~b}) . \mathrm{K}_{\mathrm{Id}}$ appears to have fallen from an initial value of over $2.4 \mathrm{MPa} \mathrm{m}^{1 / 2}$ (frame 1) to just over $1.7 \mathrm{MPa} \mathrm{m}^{1 / 2}$ (frame
7). The $\mathrm{K}_{\mathrm{Id}}$ values are subject to systematic errors, resulting mainly from the magnification change between exposures, which may be as high as $15 \%$. Nevertheless, the drop in $K_{I d}$ is significant. A plausible explanation for the behaviour is as follows. The apparently high initial toughness of the material is due to initiation from a blunt starter notch, rather than from a sharp crack. Once the crack starts to move, release waves from the crack tip act to reduce the stress intensity factor, but dynamic effects prevent this from happening instantaneously. Theoretical and numerical work by Freund (1973) and Sih (1973) showed that a step function stress pulse incident on a crack causes $K_{\text {Id }}$ to change with time, $t$, initially as $t^{12}$, before reaching the steady-state value after a time roughly equal to the time taken by a longitudinal wave to travel twice the Based on the initial notch length of $10 \mathrm{~mm}$, and a longitudinal wave speed expected response time is about $10 \mu \mathrm{s}$ for the experime exists for $\mathrm{K}_{\mathrm{Id}}$ after frame 7 , fracture surface markings showed that (Welliams, 1984). It is clear suggesting a lower bound of $1.6 \mathrm{MPa} \mathrm{m}^{1 / 2}, \mathrm{~K}_{\mathrm{Ic}}$ does indeed occur over a timescale of about then from Fig. 3 that the signifle $10 \mu$ s. It is generally accepted that the froction with velocity; the changes in $K_{\text {Id }}$ described above, 3(a)). More detailed studies on the uniqueness of the crack specd (given be carried out using the technique described in the next section.

\section{HIGH RESOLUTION MOIRE PHOTOGRAPHY}

In this section, an altemative technique for simultaneously visualising both in-plane displacement In this sectis is quasi-static displacement field around a crack tip.

The experimental arrangement is shown in Fig. 4. The basic principle is that a crossed specimen grating is imaged onto a crossed reference grating. A set of crossed linear mismatch fringes (or "carrier" fringes) is produced by choosing a magnification of slightly greater than unity. The fringes will distort as the specimen deforms, but provided the mismatch fringes have a sufficiently high spatial frequency, the $\mathrm{u}_{\mathrm{x}}$ - and $\mathrm{u}_{\mathrm{y}}$-licld fringes do not become parallel at any point in the field of view, and can therefore be subsequently separated. The required frequency of the carrier fringes the fringes can be recorded even by a camera with only limited spatial resolution.
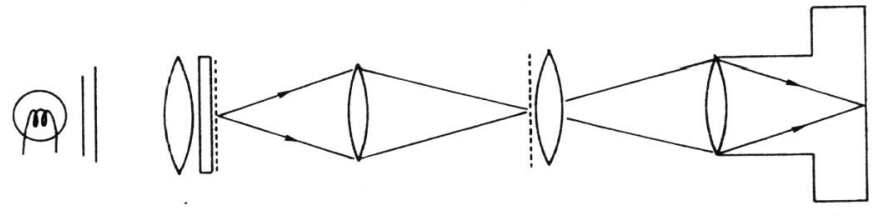

$P L \quad D \quad F \quad H_{1} \quad S G$

$\mathrm{OL}_{1}$

$\mathrm{RG} \quad \mathrm{FL}_{2}$

Fig. 4. Experimental arrangement for high resolution moire photography. PL: projector lamp; D: ground glass diffuser; F: filter (Kodak Wratten No. 44); $\mathrm{FL}_{1}, \mathrm{FL}_{2}$ : field lenses; $\mathrm{SG}$ : specimen grating; $\mathrm{RG}$ : reference recording camera with lens.

Formation of the fringes outside the camcra in this way considerably relaxes the basic trade-off Formation of the fringes outside the camcimen grating and the field of view mentioned earlier. For between the spatial frequency of the specimen grating frequency was increased to $150 \mathrm{lmm}^{-1}$ (cf. $40 \mathrm{lmm}^{-1}$ ), and the field of view was enlarged to $26 \times 36 \mathrm{~mm}^{2}$ (cf. $6 \times 10 \mathrm{~mm}^{2}$ ). A high quality objective lens $\left(\mathrm{OL}_{1}\right)$ is required to resolve $150 \mathrm{lmm}^{-1}$ at a magnification of unity. An Olympus

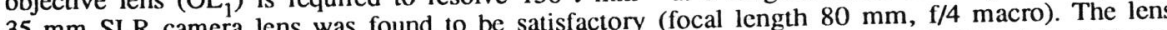
(he iris (Burch and Forno, 
1982). This reduced the effect of lens aberrations, and increased the fringe contrast. Field lens $\mathrm{FL}_{2}$ images the iris plane of $\mathrm{OL}_{1}$ onto the iris plane of objective lens $\mathrm{OL}_{2}$. The fringe contrast can $\mathrm{b}$ improved still further by replacing the reference grating with one of half the specimen spatia frequency $\left(75 \mathrm{lmm}^{-1}\right)$, and stopping $\mathrm{OL}_{2}$ down so that it collects only the diffracted beams insid the image of the mask. The interpretation of the moiré fringe pattern remains unchanged; the theory behind this modification will be published elsewhere.

Single edge notch (SEN) specimens of PMMA (dimensions $305 \times 76 \times 6 \mathrm{~mm}^{3}$ ) were used for the experiments described here. The specimen geometry is shown in Fig. 1(b). Both reference an from a He-Ne laser: created on holographic emulsion Agfa 10E 5 by two-beam interference stripping film was not sufficiently fine-grained. The drawback of the polyester is that although only $100 \mu \mathrm{m}$ thick, it inhibit crack the gratings were attached with cyanoacrylate adhesive, a groove $0.35 \mathrm{~mm}$ wide was the After machined through the polyster down to the PMMA substrate, along the expected path of the crack. In each specimen, a charp crack was initiated from the blunt starter notch with a the blade, and grown to the required length by loading the specimen at $\mathrm{K}_{\mathrm{I}} \approx 0.9 \mathrm{MPa} \mathrm{m}^{1 / 2}$. A moire fringe pattern from one of the specimens is shown in Fig. 5.

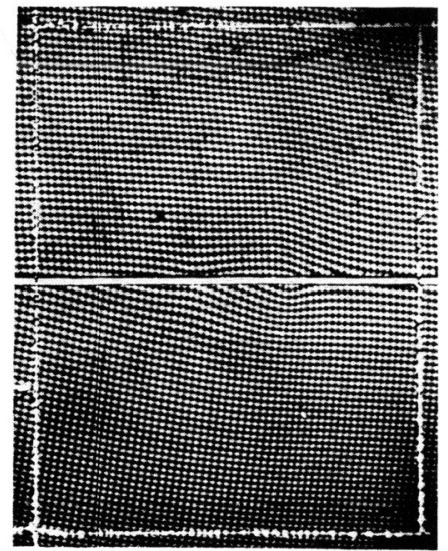

Fig. 5. Moire fringe pattem around a quasi-statically loaded crack in PMMA. The position of the scribed rectangle (dimensions $26 \times 36 \mathrm{~mm}^{2}$ ) on the specimen is shown in Fig. 1(b); the crack is in the conventiona orientation, with the tip at the centre of the rectangle. The specimen grating frequency is $150 \mathrm{lmm}$.

Computer analysis of fringe patterns

One drawback to the technique described in the previous section was the time taken to convert the information in the fringe pattems to displacement data suitable for lcast-squarcs analysis. Analysis of the seven frames, which covered an event lasting $12 \mu \mathrm{s}$, took approximately 50 man hours. It will be shown here how crossed carrier fringe patterns (such as Fig. 5) can be analysed automatically by computer.

The image processing technique is based on the Takeda Fourier transform method (Takeda et al. 1982). The original technique was applied to one set of fringes, but it also works well with two sets of crossed finges, provided the carrier frequencies of the two finge patterns are surficiently high. 2-D Fourier transformation of the fringes reveals five peaks: two for the $u_{x}$ fringes; two for the $\mathrm{u}_{\mathrm{y}}$; and one representing the d.c. term. One of the fringe peaks is isolated, with the remainder of the transform plane being set to zero; this is then followed by an inverse Fourier transform. The image is now a complex 2-D array, where the phase at any point equals the phase of the corresponding fringes, but wrapped onto the range 0 to $2 \pi$. For example, selection of the positive ${ }_{x}$ peak results in a phase map which will be denoted by $\Phi_{x}(x, y)$. Repeating the process werence iringe pattern recorded after loading the specimen results in a second phase map. The difference between the post-load and pre-load maps, $\Delta \Phi_{x}$, is shown in Fig. $6(\mathrm{~b})$. The computer unwraps the representing the $\mathrm{b}_{\mathrm{y}}$ dsplacering phending on the sign of the jump, $2 \pi$ is cither added to or subtracted from the remaining elements of the column. The process is repeated, cither added to or subtract difference phase maps have been unwrapped, the displacement components are calculated as

$$
\begin{aligned}
& u_{x}=p_{s} \Delta \Phi_{x} / 2 \pi \\
& u_{y}=p_{s} \Delta \Phi_{y} / 2 \pi
\end{aligned}
$$

. The slight asymmetry of the phase map where $\mathrm{p}_{\mathrm{s}}$ is the pitch of, the specimen grating $(6.58 \mu \mathrm{m})$. The slight asymmetry of the phase about the crack line is due to the grating not lying preciscly along the $x-y$ coordinaeld has been calculated by eqn. (4), a simple rotation matrix is therefore applied to give the true $u_{x}$ and $u_{y}$ components.

The value of $\mathrm{K}_{\mathrm{I}}$ was estimated by least-squares analysis, as in the previous section. In this case, the crack was stationary so the quasi-static form of eqn. (2) could be used. Based on the timescale of $100 \mathrm{~s}$ between applying the load and recording the photograph, Young's modulus and Poisson's ratio were taken to be $3.08 \mathrm{GPa}$ and 0.35 , respectively. The stress intensity factor resulting from the analysis of the phase maps shown in Fig. 6 was $0.490 \mathrm{MP}$
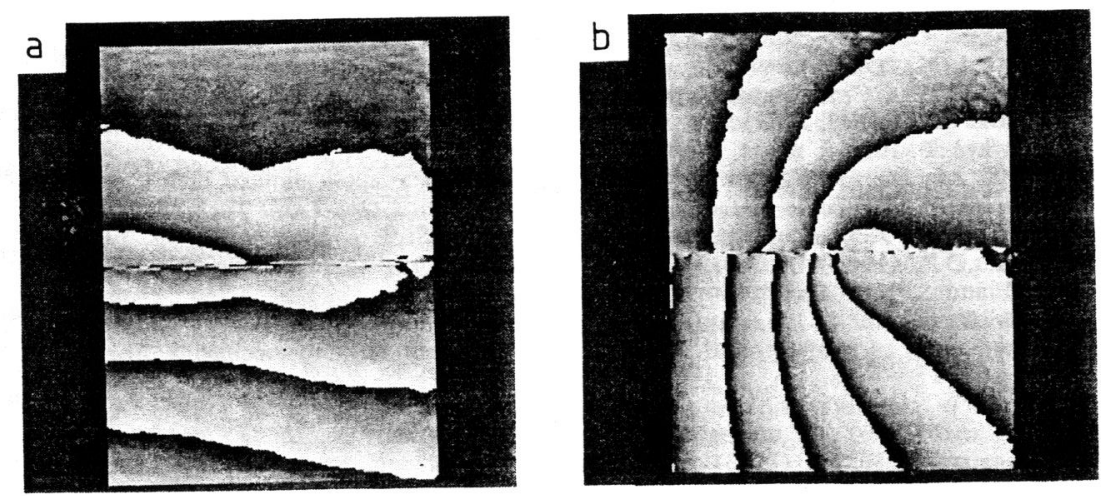

Fig 6. Difference phase maps $\Delta \Phi_{x}$ (a) and $\Delta \Phi_{y}$ (b) showing the $u_{x}$ and u displacement components resulting from mode I loading of the crack. The region covered corresponds to the scribed rectangle in Fig. S (dimensions $26 \times 36 \mathrm{~mm}^{2}$ ). The phase ranges from 0 (black) to $2 \pi$ (white); the phase discontinuities occur at intervals of $2 \pi$, representing a relative displacement equal to the specimen grating pitch, $6.58 \mu \mathrm{m}$. 


\section{CONCLUSIONS}

It has been shown how the techniques of moire photography and high speed photography can be combined to measure the in-plane displacement ficld around the tip of a fast crack. Double subsequently spatilly fitered 10 generate $u$ and $u$ field fringe pattens. $K_{I}$ was calculated by least-squares analysis of the displacement field. An $u^{x}$ mproved arrangement was proposed in which the camera records the moire fringes, rather than the specimen grating. Quasi-static tests were carried out with a specimen grating frequency of $150 \mathrm{l} \mathrm{mm}^{-1}$. The errors in stress intensity factor were estimated to be up to $15 \%$ for the dynamic experiments, reducing to $2 \%$ for the static tests with the improved apparatus.

Moiré techniques appear to offer important advantages over photoelasticity and the method of caustics. Photoelasticity requires thick specimens to achieve the necessary sensitivity; to avoid the region of triaxial stress, datapoints vell away from the crack tip are therefore used to calculate $\mathrm{K}_{\mathrm{Id}}$, and this may introduce appreciable errors when $\mathrm{K}_{\mathrm{Id}}$ is changing rapidly. In the dynamic experiments described here, all the datapoints were within $2.5 \mathrm{~mm}$ of the crack tip. The method of in-plane displan out-of-plane displacements, which are thought to respond more slowly than in-plane dicslocents method, the data processing time, is significantly reduced by the automatic fringe analysis

\section{ACKNOWLEDGMENTS}

We are grateful to N. Wilson and M. F. Turner for initial experimental work. One of us (J.M.H) was supported by Research Followships from Gonville and Caius College, Cambridge and from the Science and Engineering Research Council.

\section{REFERENCES}

Burch J. M. and C. Forno (1982). High resolution moire photography, Opt. Eng., 21, 602-614. Dally, J. W. (1979). Dynamic photoelastic studies of fracture. Exp. Mech., 19, 349-361.

and $L$. B. (1973). Crack propagation in an elastic solid subjected to general loading-III. Stress

Hu X. M., S. J. P. Palmer and J. E. Field (1984). The application of high speed photography and white light 1950ck A to ther Tech., 303-306.

Manogg P. (1964). Anwendung der Schattenoptik zur Untersuchung des Zerreissvorganges von Platten. Ph.D. Thesis, University of Freiburg.

Nishioka T. and S. N. Atluri (1983). Path-independent integrals, energy release rates, and general solutions of near-tip ficlds in mixed-mode dynamic fracture mechanics. Eng. Fract. Mech. 18 $1-22$.

Peters W. H., W. F. Ranson, J. F. Kalthoff and S. R. Winkler (1985). A study of dynamic near-crack-tip fracture parameters by digital image analysis. J. de Physique, 46, C5-631-638.

Rossmanith, H. P. (1983). Determination of dynamic stress-intensity factors by holographic interferometry, Opt. Lasers Eng., 4, 129-143.

Sciammarella C.A. (1982). The moire method- a review, Exp. Mech., 22, 418-433.

Sih G. C. (1973) Handbook of stress intensity factors, Lehigh University.

Takeda M., H. Ina and S. Kobayash (1982). Fourier transform method of fringe-pattern analysis for computer-based topography and interferometry, J. Opt. Soc. Am., 72, 156-160.

Theocaris P. S. and E. E. Gdoutos (1972). An optical method for determining opening-mode an edge sliding-mode stress intensity factors. ASME J. Appl. Mech., 39, 91-97.

Yang W. and L.B. Freund (1985). Transverse shear effects for through-cracks in an elastic plate.

Int. J. Solids Struct. 21 977-994. 